\title{
Impact of the soil resistivity on IR drop when applying a cathodic protection system with galvanic anodes on PCCP
}

\author{
A. Mujezinović \& A. Muharemović \\ Faculty of Electrical Engineering, University of Sarajevo, Bosnia and \\ Herzegovina, Sarajevo, Bosnia and Herzegovina
}

\begin{abstract}
This paper analyzes the phenomenon of potentials deviation (ON/OFF potentials) as a result of changes in the soil resistivity, when applying cathodic protection systems with galvanic anodes on PCCP (pre-stressed concrete cylinder pipes) in ambient desert conditions. Measurement of ON and OFF potentials on a long section with different values of the soil resistivity have shown that there are great deviations in parts of sections which are proportional to the measured values of the soil resistivity. Analyzing the changes in a value of IR drop show that the ON potential changes very little in the case of high values of the soil resistivity, as well as that on these sections, a high value of IR drop appear, i.e. great change OFF potential. Over time, this difference between the ON and OFF potentials in these sections decreases and this is a case of a full polarization of PCCPs. If the value of the electrical soil resistivity is relatively low, it can be concluded that the IR drop is negligible.

Keywords: cathodic protection (CP), protection current, measurements, desert conditions, ON/OFF potentials, pre-stressed concreted cylinder pipe (PCCP).
\end{abstract}

\section{Introduction}

Efficiency of the applied cathodic protection system on a concrete underground metal object can be evaluated by recording (measurements - recording) potential values before and after its polarization. It involves measuring (recording) OFF (true potential) and ON potential in all relevant points of the object that is the being protected from the corrosion. Due to the object's configuration, the most 
common situation is that it is not possible to make a realistic and credible measurement of both previously mentioned potential values $[1,2]$.

When registering ON potential of metal object, it must be kept in mind the fact that this potential is composed of components (which are normally components of the systematic errors of measurement).

Natural (stationary) potential,

polarization, and

IR drop as a measurement error.

Standard $\mathrm{Cu} / \mathrm{CuSO}_{4}$ saturated reference electrode (CSE), each with its resistance to the electrolyte, is typically used with this kind of measurements. This resistance is directly a function of the value of specific resistance of electrolyte (soil resistivity). In all serious measurements of polarization level of metal object, the IR drop, that registered by measurement and that presents error, should be eliminated. Measurement error, which does not take into account the IR drop, can create wrong impression of the real level of polarization of the object. The easiest way to eliminate this error is to record ON/OFF potentials of the object.

In the case of application of cathodic protection systems of metal object, during its polarization, its potential changes over time (object polarizes). In this paper the authors have analyzed the changing relationship ON/OFF potentials at all stages of polarization PCCP which is being protected by $\mathrm{Zn}$ galvanic anodes. In doing so, the fact that some places have low and some high values of soil resistivity was taken into account.

Based on measurements of potential, it can be stated that measurement error (IR drop) is more evident in the polarization phase of the object. After full polarization of the object, this error is treated as a systematic error, which is thereby also defined.

The goal of this paper is to determine the objectivity of standards of cathodic protection "shift" potential (decay) in the interest of protecting the object, based on long-term measurements recording of ON/OFF potentials of protected object.

\section{Potential measurement background}

The basic criterion for assessing the functionality of cathodic protection system is based on measuring the potential of the protected object relative to a reference electrode. When measuring (recording) the potential, a milivoltmetar with high internal resistance is used, thus with the aim of eliminating the current that could flow in the circuit reference electrode - soil - protecting object - milivoltmetar. The current flow in protection circuit could polarize the reference electrode, or change its own potential [3-5].

Due to its dimensions, the reference electrode cannot physically be placed on the surface of protected object, and it is always located at some distance. The shortest distance between the reference electrode and the protected object is the depth of object burial in the ground (as shown in fig. 1). 


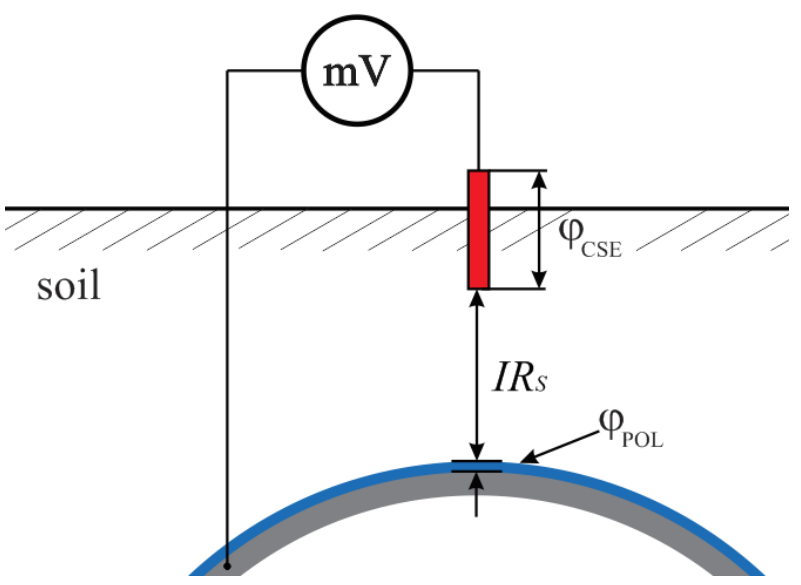

Figure 1: $\quad$ Impact of IR drop on protection potential measurement.

The distance between the surface of the protected object and the reference electrode results in the generation of voltage drop in the ground, that is IR drop, which is included in milivoltmeter showing. In this case, the voltmeter will register a potential difference which is equal to $\mathrm{ON}$ potential and can be expressed as follow:

$$
\varphi_{O N}=\varphi_{O F F}+I R_{S}+\varphi_{C S E}=\varphi_{C O R C}+\varphi_{P O L}+I R_{S}+\varphi_{C S E}
$$

where $\varphi_{O N}$ is measured ON potential, $\varphi_{O F F}$ is protected objects OFF potential, $I R_{S}$ is IR drop in the soil, $\varphi_{C S E}$ is potential of reference electrode, $\varphi_{C O R C}$ is free corrosion potential of the pipeline (cathode), $\varphi_{P O L}$ is polarization potential of pipeline.

Voltage drop in the soil (IR drop) can be calculated as:

$$
I R_{S}=\rho \cdot j \cdot d
$$

where: $\rho$ - soil resistivity $(\Omega \mathrm{m})$;

$j$ - protection current density $\left(\mathrm{A} / \mathrm{m}^{2}\right)$;

$d$ - the distance between the pipeline and the reference electrode (m).

As for the longer objects such as pipelines, it is necessary to measure the potential over its full length. For the case of recording only ON potential, due to the longitudinal changes in the soil resistivity, wrong conclusions about the potential existence of corrosion could be reached [6]. In order to obtain a complete picture of the protection functionality, it is necessary to carry out simultaneous recordings of ON/OFF potential.

In eqn (2) it is evident that the IR drop is a function of distance between the reference electrode (CSE) and the object, the soil resistivity and the density of 
the protection current. In order to eliminate the IR drop, it is necessary to affect on at least one of the previously mentioned functional dependences.

Today, the most commonly used method to eliminate IR drop is current interrupted method. The method is based on the fact that at the time of protection current breaking, voltage drop in the ground is almost instantly eliminated. When implementing this method, it is necessary to ensure prevention of depolarization of protected object. Practice has shown that uninsulated steel constructions depolarize to $50 \mathrm{mV}$ within the interval of $0.1 \mathrm{sec}$. Therefore, measuring of OFF potential must be performed in less than $0.1 \mathrm{sec}$, so the measurement error resulting from depolarization is within acceptable limits.

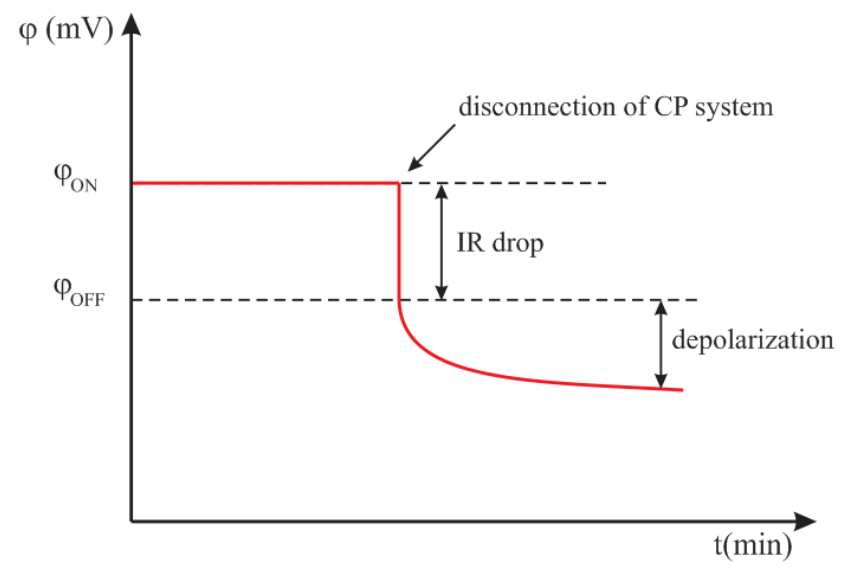

Figure 2: The time-dependent depolarization of the protected object.

Fig. 3 presents one of the most commonly used techniques for measuring of ON/OFF potentials, i.e. CIPS (Close Interval Potential Survey) techniques. By this method, the potential is being measured, so that the positive end of the milivoltmeter is connected to the pipeline (at fixed measuring points), while the negative end of a voltmeter connected to the transferable reference electrode. The measurement is performed by a portable electrodes placed on the ground surface, at intervals of approximately 1 meter, along the pipeline section. Measuring and recording of pipeline potential is implemented on a large number of points, with short distance among them, so that it can be considered as continuous potential recording. Whit such proceeding, ON potential is recorded, that is the one that contains the IR drop. In order to get OFF potential of protected object, systematic error of measurement that results from the existence of IR drop in soil should be eliminated. The most practical way to eliminate these systematic errors, in case of performing of such intensive measurements, is the current interrupted methods. This is achieved by temporary synchronized turning off/on of cathodic protection systems using current interrupter. Measured potentials in this case, have no IR drop because the current intensity is equal to zero. 


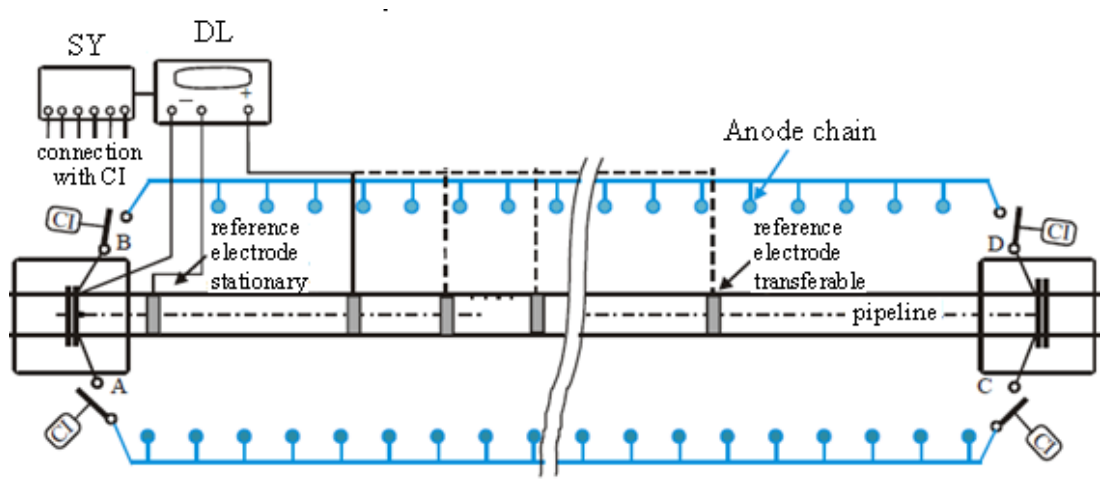

Figure 3: The principle scheme of CIPS recording(DL - data logger, SI device for synchronization, $\mathrm{CI}$ - current interrupter)

\section{Analysis of measured results}

Recording ON/OFF potentials and natural potential was performed on sections of PCCP with large diameter app. $4 \mathrm{~m}$. The average burial depth of considered PCCP is $3 \mathrm{~m}$. PCCP is protected by cathodic protection system with galvanic anodes of zinc, which total length is $6.4 \mathrm{~m}$. The object is placed in the desert ambient conditions.

In figs 4 and 5 , the diagrams of the protective potential changes and the soil resistivity, respectively, are shown. With the given diagram it is evident that at high levels of the soil resistivity, potential changes are lower. This stems from

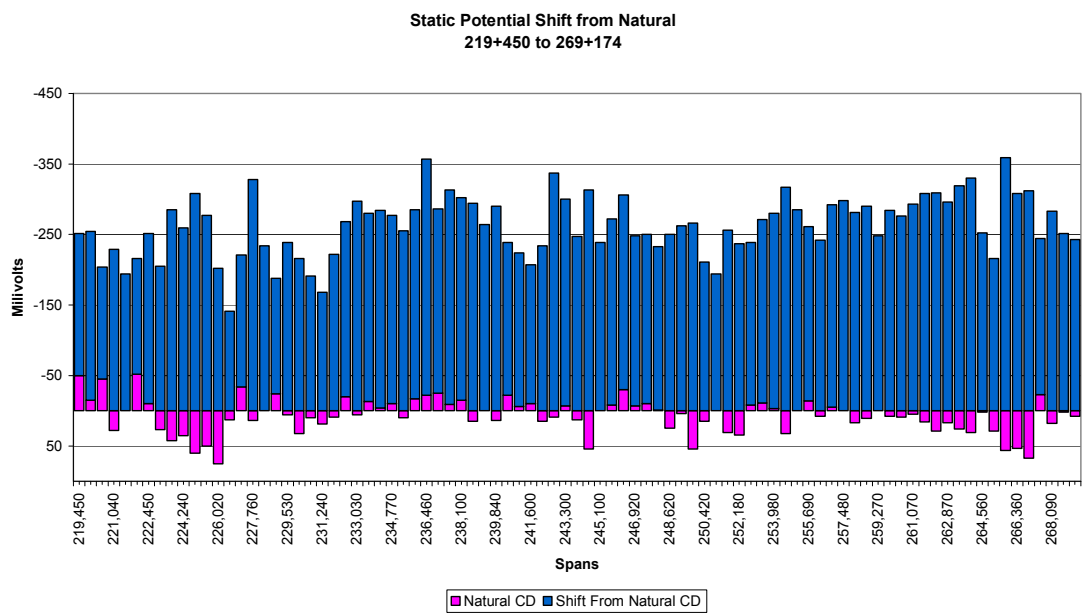

Figure 4: $\quad$ Static potential shift from natural potential. 


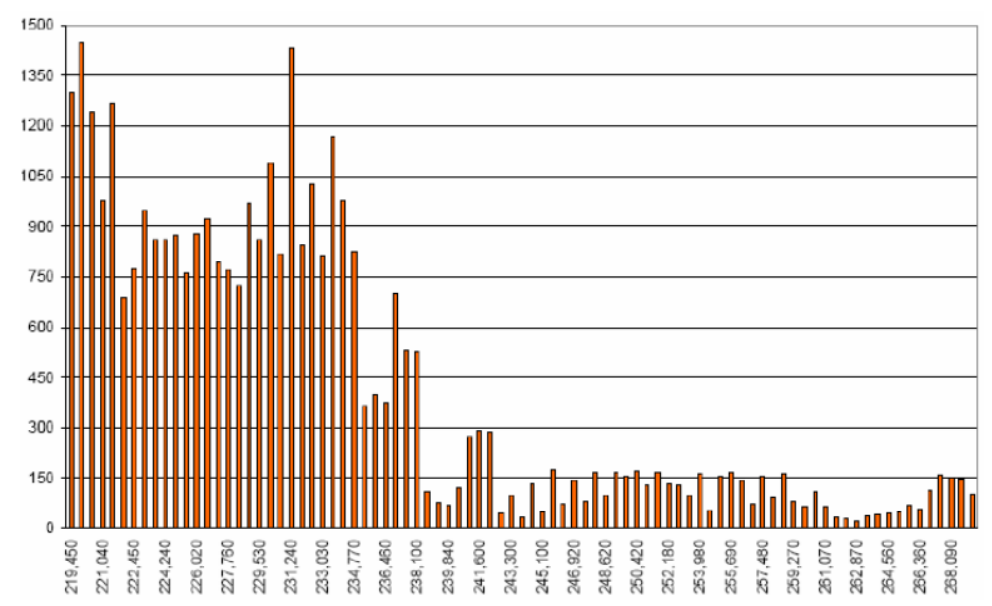

Figure 5: $\quad$ Value of the soil resistivity.

the fact that in the prevailing conditions establishes a lower value of protective current density circuit cathodic protection systems due to the higher value of the total polarization resistance.

In fig. 6, the diagram of recorded ON/OFF potentials of PCCP buried in the soil with high value of electrical resistivity is shown.

TSTB $156+030$ to $156+630$

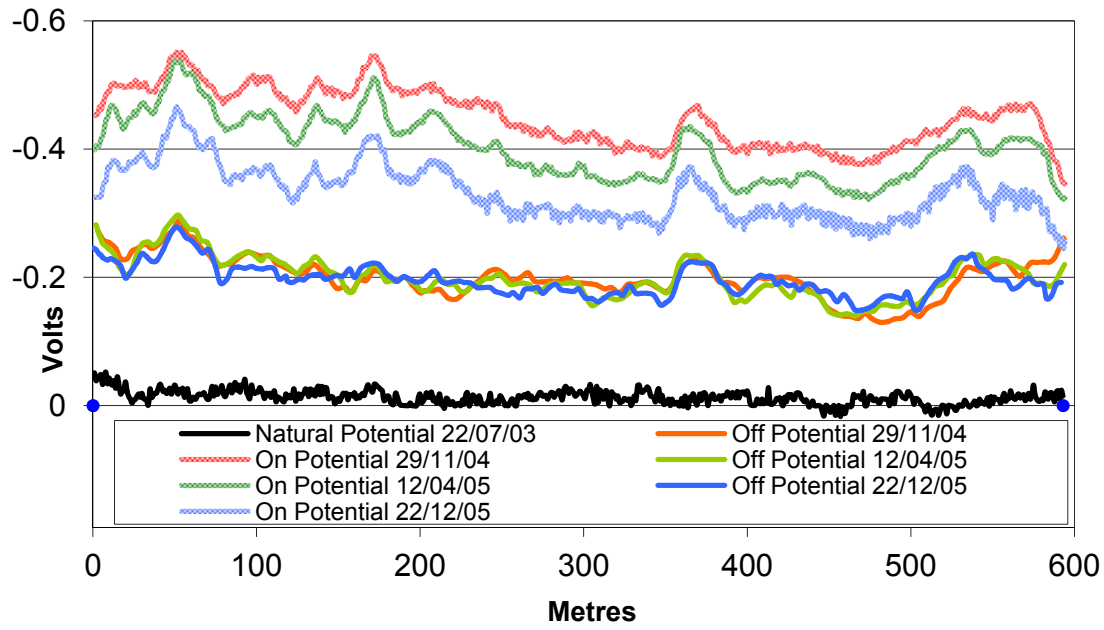

Figure 6: Diagram of the ON/OFF potentials at section where high values of soil resistivity are measured. 
In fig. 7 a diagram of recorded ON/OFF potentials of PCCP buried in the soil with low value of electrical resistivity is shown.

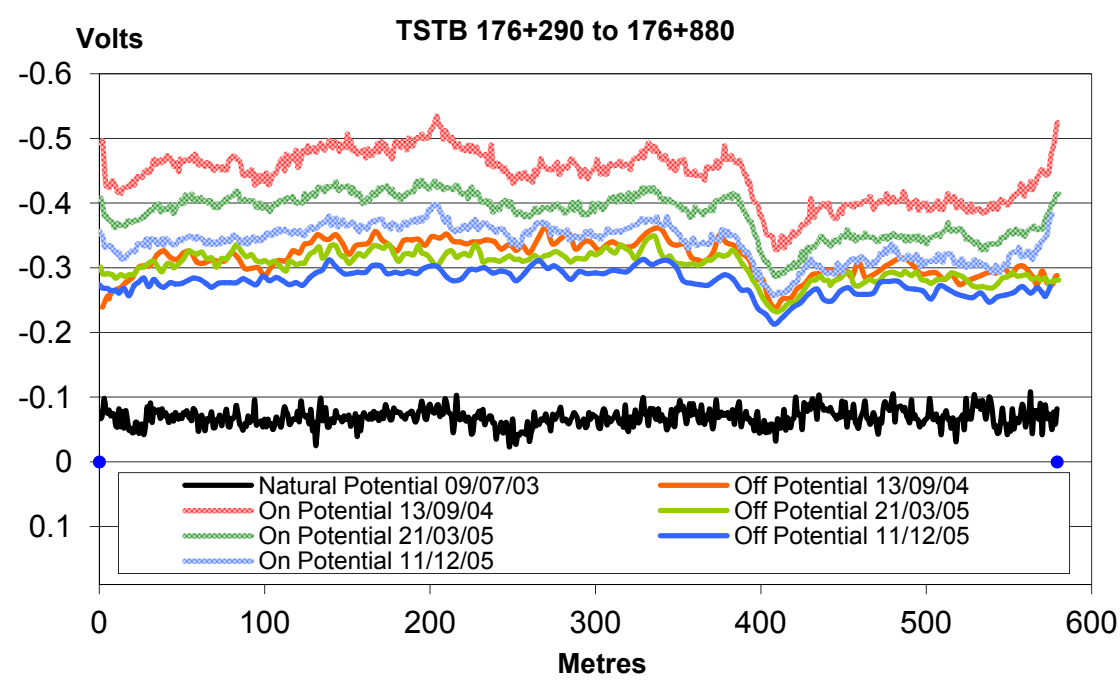

Figure 7: Diagram of the ON/OFF potentials at a section where the low values of soil resistivity are measured.

It is evident that in both cases, the ON potential is fairly unified, while the OFF potential is significantly lower in a case of low soil resistivity (closer to the ON potential), therefore in this case and the IR drop is much lower [7-9]. During operation of cathodic protection systems, increases of groundbed resistivity and polarization resistance of PCCP resulting in a decrease of total current in the circuit, decreases the ON/OFF potentials and reducing the IR drop.

\section{Atypical cases (anomalies on sections)}

Fig. 8 shows one atypical case of changes of the IR drop along the section where are measured longitudinal changes in the soil resistivity. At this section of PCCP, ON potential remains fairly constant while the OFF potential positive reading, or when OFF potential weighs to natural potential. Precisely in these areas IR drop in the soil increases. The characteristic section is St. No. 200+000 to St. No. 200+520 (between 300 and $500 \mathrm{~m}$ ).

On the part of sections from 300 to $500 \mathrm{~m}$, OFF potential becomes more positive and closer to the values that are closer to its natural potential. In this part of the section, applied cathodic protection system becomes less efficient. ON potential remains approximately constant, therefore, it can be concluded that the IR drop is increased. In this part of the section it was registered the increase of electrical soil resistivity and the decreases of protective current density. 


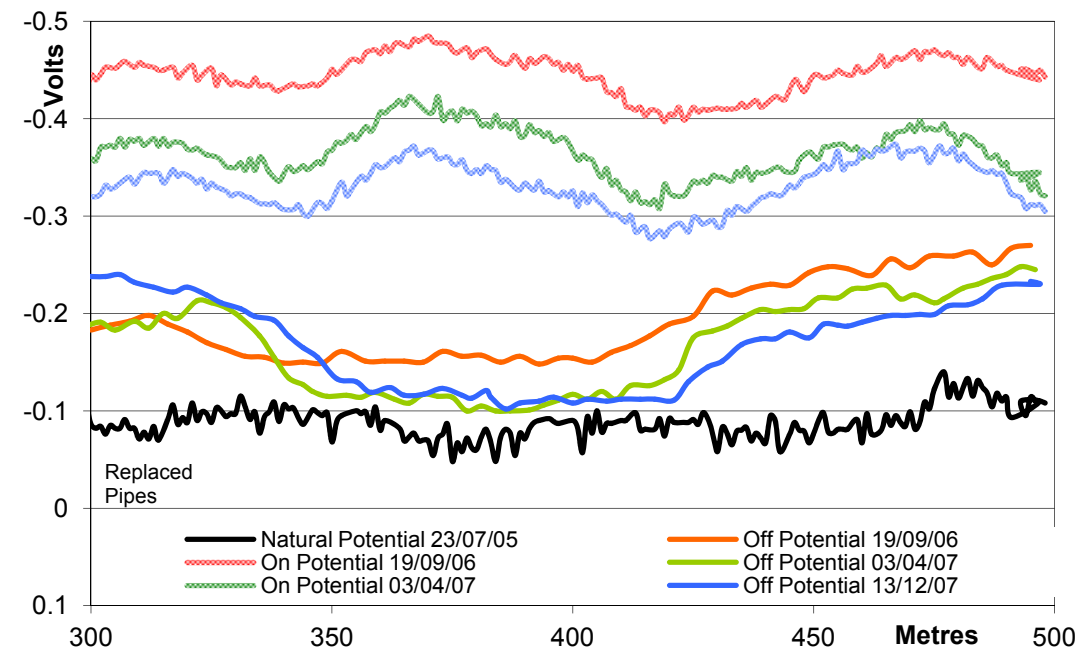

Figure 8: CIPS recording for section St. No. $200+000$ to St. No. $200+520$.

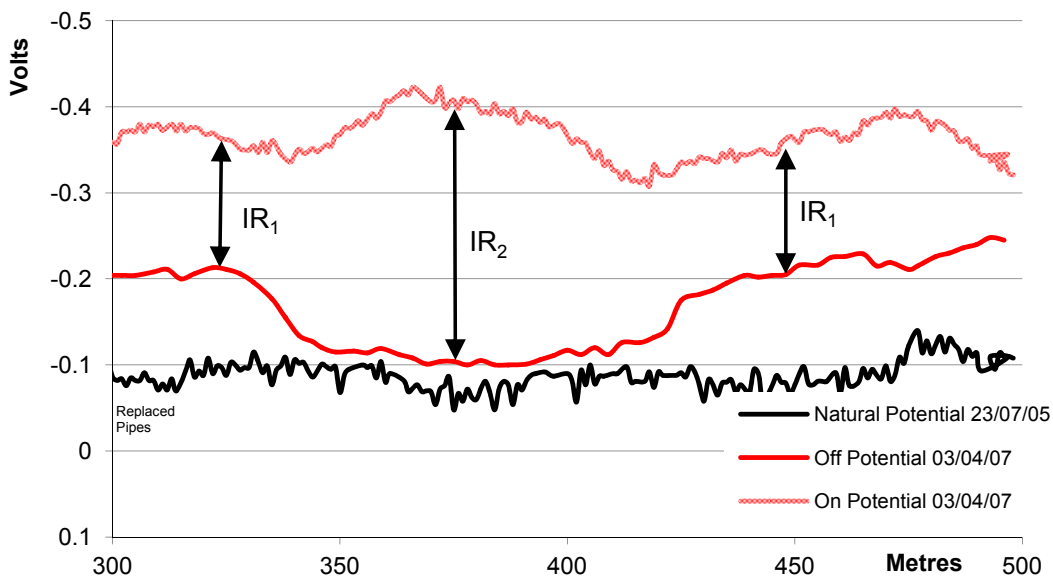

Figure 9: $\quad$ Anomalies on Section St. No. $200+000$ to St. No. $200+520$.

\section{Conclusion}

The measured values of the potential always contain the IR drop of the potential in the soil. This component is eliminated mainly temporary by switching off the system of cathodic protection. Potential difference ON/OFF defines the value of the IR drop. Finding the exact value of OFF potential has great practical importance for the assessment of efficiency of the applied cathodic protection 
system, and also depends on the level of polarization as well as the value of soil resistivity.

When analyzing the IR drop, it can be concluded that:

After turning off cathodic protection system, IR drop currently disappears (remains polarization component which eventually disappears). Because of this fact, when recording the OFF potential, locations measuring electrode is not so important.

- In order to accurately determine the value of the potential drop at the border pipeline-ground, time between the turning off moment of cathodic protection and measuring moment should be as short as possible.

- If the pipeline is polarized for a long time, more time is needed for depolarization of the object.

\section{References}

[1] C. Qui, Model for interpretation of pipeline survey data, Ph. D. Dissertation, University of Florida, Gainesville, FL, 2003.

[2] F. Dawalibi \& R. D. Southey, Computer Modeling of Pipelines and Their CP Systems for IR Drop Measurement Correction, NACE International, CORROSION 98, March 22-27, San Diego Ca., 1998.

[3] L. Lazzari, \& P. Pedeferri, Cathodic Protection, Polipress, Milano, 1st edition, 2006.

[4] A. Muharemović, I. Turković, A. Kamenica, Basic specifics for the assessment of Zinc anode strings for cathodic protection, $13^{\text {th }}$ International expert meeting Power Engineering, Maribor, Slovenia, 2004.

[5] NACE Standard RP 0187, Design Considerations for Corrosion Control of Reinforcing Steel in Concrete, NACE Int., Houston, TX, 2000.

[6] M.E. Parker, \& E.G. Peattie, Pipeline Corrosion and Cathodic Protection, Third Edition, Gulf Publishing Company, Houston, Texas, 1984.

[7] Energoinvest Sarajevo, External cathodic protection system - scope of work, 19051-S-395- 10-MC-0028-00, Sarajevo, 2006.

[8] Energoinvest Sarajevo, Potential survey procedure, 19051-S-395-10-MC0028-00, Sarajevo, 2006.

[9] Energoinvest Sarajevo, External Cathodic Protection Project for PCCP, Operation \& Maintenance period final report, 19051-S-395-10-MC-0028$00,2006$. 